\title{
The effect of rapid maxillary expansion on nasal aimway resistance
}

\author{
Dale V. Hartgerink, D.D.S., M.S.," Peter S. Vig, B.D.S., Ph.D., D. Orth., F.D.S.R.C.S., ${ }^{\star \star}$ and \\ Diana Wolf Abbott, D.D.S., M.S.*** \\ Ann Arbor, Mich.
}

The purpose of this study was to evaluate changes in nasal resistance to airflow in persons undergoing rapid maxillary expansion and to reevaluate the responses at a 1-year follow-up. Nasal resistance measurements, assessed in four modes (natural state, anterior nares dilation with Tygon tubing, following administration of decongestant, and nares dilation with tubing and decongestant), were taken on a group of 38 patients receiving rapid maxillary expansion and compared with a control group not receiving expansion. Thirty-three of the patients were reevaluated 9 to 12 months after expansion was completed. Eighteen subjects in the control group were also reevaluated. Oral/nasal aifflow rates (percent nasality) were recorded for the control group and for some of the expansion patients. Results indicated that some subjects receiving rapid maxillary expansion had a significantly higher nasal resistance than the control group. There was a significant median reduction in nasal resistance following rapid maxillary expansion, measured in the natural state only, and this appeared to be stable up to 1 year after maximum expansion was obtained. Rapid maxillary expansion appeared to effect an expansion at the anterior nares, which contributes to nasal resistance reduction. Individual variation in nasal resistance values was considerable and hence the median response for the group was not a reliable estimate of individual response. Due to the high individual response variability, rapid maxillary expansion is not a predictable means of decreasing nasal resistance.

(AM J ORthod Dentofac ORTHOP 1987;92:381-9.)

$\mathbb{E}_{\text {xpansion of the midpalatal suture has be- }}$ come an accepted procedure for the treatment of maxillary constriction and associated arch length discrepancies. The concept of maxillary expansion has been extended to the nasal cavity as previous studies have suggested that with expansion, increases in nasal width and volume are obtained. ${ }^{1.4}$ Thus, it is commonly held that maxillary expansion diminishes resistance to nasal airflow.

Controversy over the role of respiration in the cause of malocclusion has spurred interest in the use of rapid maxillary expansion (RME) to enhance nasal respiration. In particular, the "long-face syndrome" or "adenoid facies" has been assumed to originate from mouth breathing due to high nasal resistance to airflow. ${ }^{5-7}$ Other studies disputing this have indicated that impaired

From the Department of Orthodontics, School of Dentistry, University of Michigan.

This study was supported by NIH/NIDR Grant DE-06881 and the LeGro Fund. University of Michigan

*Major, U.S. Air Force, Dental Corps. Currently in practice at Anderson Air Force Base, Guam.

**Professor and Chairman, Department of Orthodontics, University of Michigan.

${ }^{* * *}$ Clinical Instructor, University of Michigan. respiratory function can be found in patients with a variety of facial types. ${ }^{8-11}$ Facial characteristics associated with mouth breathing have been stated to include a retrognathic mandible, proclined maxillary incisors, high V-shaped palatal vault, a constricted maxillary arch, flaceid and short upper lip, flaccid perioral musculature, and a somewhat dull appearance resulting from a constant open-mouthed posture. ${ }^{12}$

To date, attempts to establish a cause-and-effect relationship between nasal obstruction and dentofacial form have proved to be equivocal. Normal nasorespiratory function has not been adequately defined and, despite claims to the contrary, the extent to which dentofacial form may be influenced by respiratory mode is unclear. The controversy has been largely due to the inability to quantify nasal obstruction and to determine the mode of breathing objectively.

Yet another problem impeding the objective study of the respiratory effects of RME is the lack of documented correspondence between increments of dental arch expansion and the associated changes in the minimum effective cross-sectional area of the nasal airway. Arch expansion is the sum of dentoalveolar movement, which may comprise both tipping and bodily displacement, and the orthopedic relocation of components of 


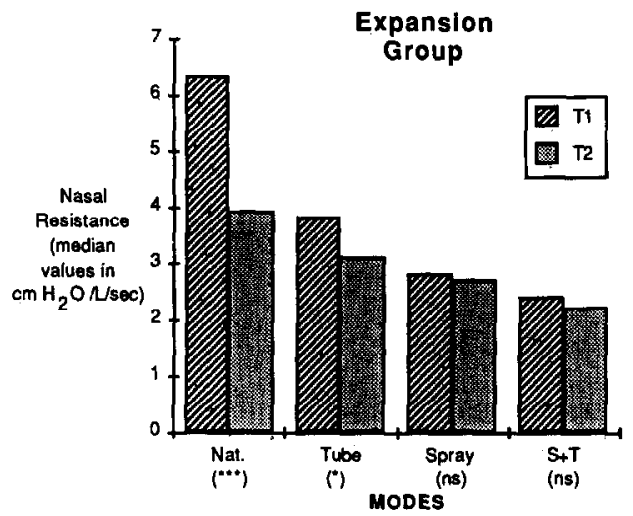

Fig. 1. Median nasal resistance for the expansion group: T1 to T2. Note: medians are displayed but statistical significance levels are based on ranking of subjects in each group. Therefore, the median values may appear to be misleading. " $P$ " values for nonparametric statistical tests are shown $\left({ }^{*}=P<0.05\right.$; $\left.{ }^{\star *}=P<0.01 ;{ }^{* \star *}=P<0.001\right)$.

the nasal cavity. Although it is relatively easy to measure intermolar or intercanine width changes, these cannot be extrapolated to yield valid information on airway dimensions. The tortuous configuration of the airway and the superimposition of structures render posteroanterior cephalograms suspect for mensuration of the area of greatest constriction in the nose.

Most, if not all, authors fail to consider the correlation among "mouth breathing," nasal resistance, and actual measured oral/nasal airflow rates. The "lips apart posture," "adenoid facies" appearance, and nasal resistance values cannot in themselves be used to adequately assess breathing as being totally nasal, oral, or something in between. Despite this, both diagnostic and treatment recommendations are often made on the basis of these criteria.

According to Warren, ${ }^{12}$ clinical impressions and patients' subjective impressions of nasal airway impairment do not consistently correlate with objective measures of airway resistance. Much of the confusion is caused by the lack of a precise definition of "mouth breathing:" Does this equate with total absence of nasal breathing or is it a combination of oral and nasal breathing with the oral component predominating? Is there a critical upper limit of nasal resistance for any individual that causes a switch from predominantly nasal to predominantly oral breathing? Is there a causal association between oral respiration and growth pattern? These are questions that need to be resolved if precise and clinically useful concepts are to be developed.

Procedures such as nasopharyngeal surgery, allergy treatment, and RME continue to be advocated to eliminate the effects of nasal obstruction on facial form.

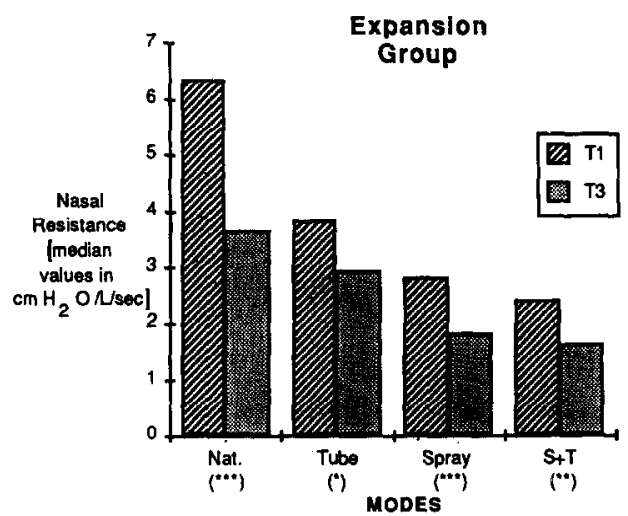

Fig. 2. Median nasal resistance for the expansion group: T1 to $\mathrm{T3}$.

RME has been advocated by some ${ }^{2,5,13}$ for both dental and rhinologic purposes in the belief that clinically significant reductions in nasal resistance to airflow occur predictably.

Several investigators ${ }^{2,3,5,14,15}$ have studied the effects of RME and reported decreases in nasal resistance following treatment. Wertz, ${ }^{14}$ however, could not justify expansion unless an obstruction was present in the anteroinferior aspect of the nose, the area most affected by maxillary expansion. Following RME, Hershey, Stewart, and Warren ${ }^{15}$ found a significant mean decrease $(45 \%)$ in nasal resistance. Their patients were reevaluated 1 year following treatment ${ }^{16}$ and the decreases in nasal resistance were found to be stable.

The purpose of our study was to measure the nasal airway resistance of patients before and after RME, and to reevaluate the response 9 to 12 months after treatment. Our specific aims were to address the following questions:

1. Does RME contribute to a decrease in nasal resistance? Are differences still apparent 9 to 12 months after treatment?

2. How variable is individual response to treatment?

3. How may clinicians predictably identify those patients who might benefit from RME in terms of reductions in nasal resistance?

\section{MATERIALS AND METHODS}

The treatment sample consisted of 38 patients who were to have RME for orthodontic indications. Pressure/flow data were obtained for these persons just before (T1) and within 1 week following RME (T2). This group ranged in age from 7.5 to 22.33 years with a median age of 11.75 years. Thirty-three of these patients were reevaluated 9 to 12 months following ex- 


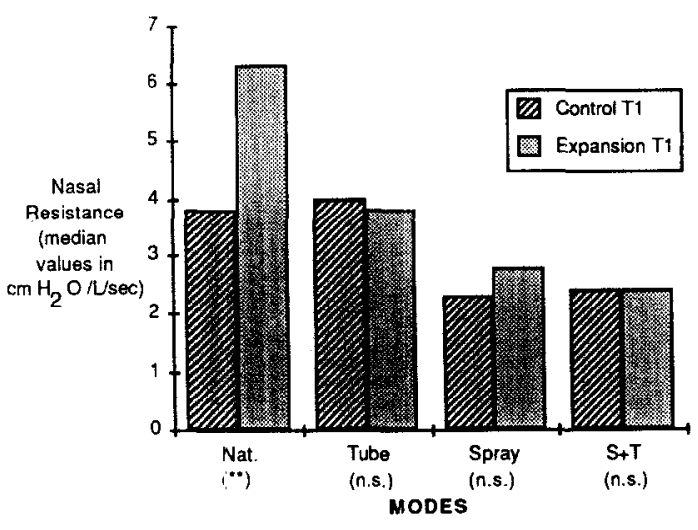

Fig. 3. Median nasal resistance compared for the control group (T1) and expansion group (T1).

pansion (T3). No patients were excluded on the basis of age and RME was accomplished with either a banded or bonded expansion device. A control group of 24 subjects was also tested. This group ranged in age from 8.3 to 14.7 years with a median age of 12.00 years. Eighteen of these subjects were reevaluated at $\mathrm{T} 3$.

Resistance determination was performed using the Perci IIC.* The Simultaneous Nasal and Oral Respirometric Technique (SNORT) apparatus, first described by Gurley and $\mathrm{Vig}^{17}$ and subsequently modified by Keall and Vig, ${ }^{18}$ was also used to obtain oral and nasal airflow values for the control group (T1, T3), and for some of the subjects in the expansion group (T3 only). The calculation of nasal resistance during nasal respiration was determined from the parameters of pressure and airflow as described by Warren. ${ }^{19}$ Resistance values were obtained at a flow rate of $250 \mathrm{cc} / \mathrm{sec}$.

For subjects tested with SNORT, continuous monitoring and recording of airflow through the nose and mouth during inhalation and exhalation were performed. Nasal respiratory pressure/flow data (nasal resistance) were recorded with both the Perci IIC and SNORT for the control group. This was done to verify that the nasal resistances obtained were comparable for the two instruments.

Four experimental conditions (modes) were used for both resistance and oral/nasal airflow determinations:

1. Natural (normal) respiration

2. Respiration with dilation of the nares using Tygon tubing of appropriate size

3. Respiration 5 minutes after administration of a nasal decongestant spray $(0.25 \%$ Neo-Synephrine hydrochloride)

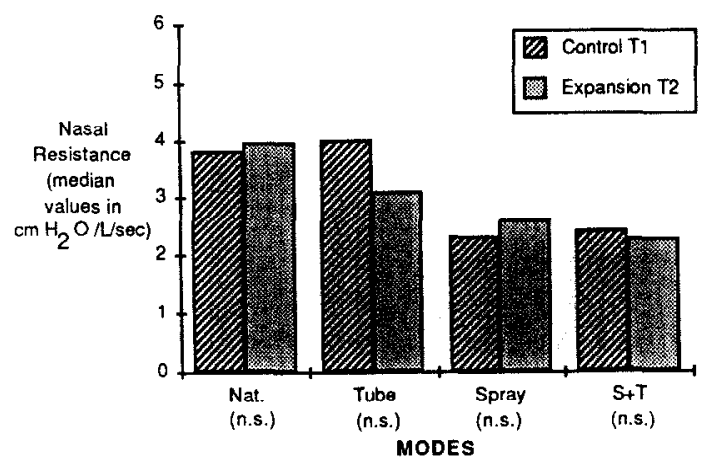

Fig. 4. Median nasal resistance compared for the control group (T1) and expansion group (T2).

4. Respiration following use of the nasal decongestant combined with nares dilation

\section{Statistical considerations}

Both the expansion and control groups were skewed with respect to nasal resistance values. For this reason nonparametric tests (Wilcoxon's signed rank test and Mann-Whitney rank sum test) were used to make comparisons within and between each sample and among the three time periods to test the hypothesis of no treatment response. An alpha level of 0.05 was set and Bonfcronni's corrcction for statistical significance was applied when appropriate (where multiple comparisons were made).

\section{RESULTS}

Comparisons are presented between groups (treatment versus control), over time, between subgroups, and for all four experimental conditions. Intra-individual variation for nasal resistance was high for all modes and time periods tested, and averaged approximately $\pm 0.5 \mathrm{~cm} \mathrm{H} 20 / \mathrm{L} / \mathrm{sec}$ for the entire group. This should be emphasized because some of the differences seen between modes or between time periods were within the range of individual variation and thus should not be considered as representing clinically or physiologically significant changes.

\section{Differences in nasal resistance within each time period}

For the expansion group, significant reductions in nasal resistance were obtained (for all three time periods) when comparing the natural state to the tubeassisted, spray- (decongestant) assisted, or spray- and tube-assisted modes. For the control group, significant reductions in nasal resistance were also seen with the spray-assisted, and spray- and tube-assisted modes at T1 and T3. However, reductions were significant with 


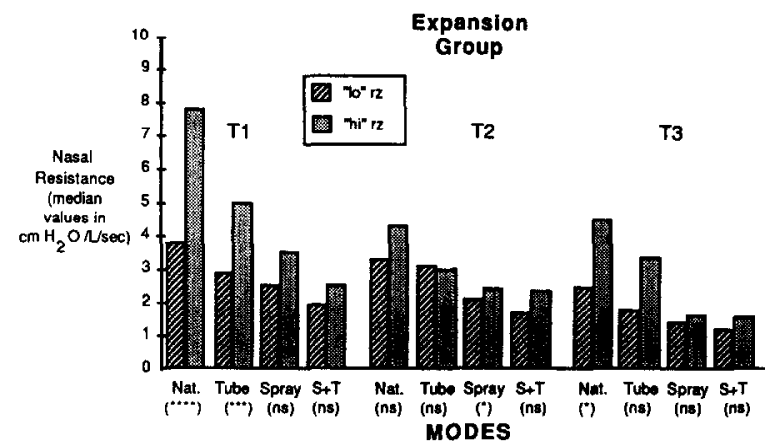

Fig. 5. Median nasal resistance for the expansion group: "lo" versus "hi." Subgroups are compared at T1, T2, and T3.

the tube-assisted mode only at T3. Nasal resistance for this group actually increased slightly at $\mathrm{T} 1$ with the use of tubes. For both treatment and control groups, the use of spray and tubes together did not significantly reduce nasal resistance more than tubes or spray alone within each time period.

\section{Differences in nasal resistance among time periods}

Significant decreases in nasal resistance were found from $\mathrm{T} 1$ to $\mathrm{T} 2$ only for the natural state and for the tube-assisted mode. See Fig. 1. Interindividual variation was very high for all modes tested. Since intraindividual variation averaged $\pm 0.5 \mathrm{~cm} \mathrm{H} 20 / \mathrm{L} / \mathrm{sec}$, decreases or increases in nasal resistance of this magnitude cannot be considered significant. Therefore, significant decreases in nasal resistance were found for $65 \%$ of the subjects for the natural state, $56 \%$ for the tube-assisted mode, $39 \%$ for the spray-assisted mode, and $35 \%$ for the spray- and tube-assisted mode. This also means that for the above four experimental conditions, $35 \%, 44 \%$, $61 \%$, and $65 \%$ of the subjects, respectively, did not show significant decreases (and may in fact have had increases) in nasal resistance following expansion. This variability is an indication of the unpredictable response on an individual or clinical basis.

Significant decreases were found in all modes from $\mathrm{T} 1$ to $\mathrm{T} 3$. See Fig. 2. Inter-individual variation was also high between $\mathrm{T} 1$ and $\mathrm{T} 3$, although not as great as that found between $\mathrm{T} 1$ and $\mathrm{T} 2$. This represents significant decreases in nasal resistance for $72 \%$ of the subjects in the natural state, $56 \%$ for the tube-assisted mode, $63 \%$ for the spray-assisted mode, and $50 \%$ for the spray- and tube-assisted mode. From T2 to T3, statistically significant decreases in nasal resistance were found only for the spray-assisted and spray- and tube-assisted modes.

Inter-individual variation in the control group was

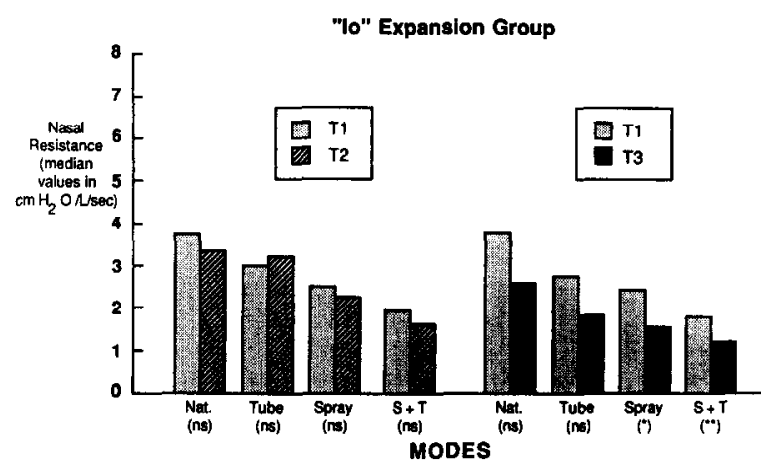

Fig. 6. Median nasal resistance for the "lo" expansion subgroup: $\mathrm{T} 1$ to $\mathrm{T} 2, \mathrm{~T} 1$ to $\mathrm{T} 3$.

also high from $\mathrm{T} 1$ to $\mathrm{T} 3$ and significant decreases were seen for the tube-assisted, spray-assisted, and sprayand tube-assisted modes, but not for the natural condition.

\section{Comparison of the expansion and control groups}

Nasal resistance values for the expansion group at T1 showed a significant difference from resistance values for the control group at $\mathrm{T} 1$ only for the natural state. A comparison of resistance values for the expansion group following the expansion at $\mathrm{T} 2$, with the values of the control group at T1, showed no significant differences between the two groups. See Figs. 3 and 4. At T3 there were also no significant differences between the groups.

\section{Identification of two subgroups in the expansion group}

Two subgroups were identified within the expansion group at T1 for nasal resistance in the natural state. A "lo" resistance subgroup comprised subjects with initial resistances less than or equal to $5.5 \mathrm{~cm} \mathrm{H20/L} / \mathrm{sec}$ and a "hi" resistance subgroup comprised subjects with initial resistances greater than $5.5 \mathrm{~cm} \mathrm{H} 20 / \mathrm{L} / \mathrm{sec}$.

A. Comparison of "lo" and "hi" subgroups within each time period. At the initial time (T1), there was a significant difference between the "lo" and "hi" subgroups for only the natural state and for the tubeassisted mode. The two subgroups were not significantly different for either the spray-assisted or sprayand tube-assisted modes. After maximum expansion (T2), the differences between the "lo" and "hi" subgroups were not significantly different for either the natural state, tube-assisted, or spray- and tube-assisted mode. However, there was a moderately significant difference between the subgroups for the spray-assisted mode, with the "hi" subgroup remaining somewhat 
higher than the "lo" subgroup. At the follow-up period (T3), differences between the two subgroups were only significantly different for the natural state, with the nasal resistance for the "hi" subgroup remaining above that of the "lo" subgroup. See Fig. 5.

B. Comparison of "lo" and "hi" subgroups between time periods. For the "lo" subgroup, there was no significant difference found in nasal resistance for any mode tested from $T 1$ to $T 2$. Also from $T 1$ to $T 3$, this subgroup did not show significant differences in nasal resistance for the natural state or tube-assisted modes, but did exhibit significant decreases in the spray-assisted and spray- and tube-assisted modes. See Fig. 6.

For the "hi" subgroup, a significant decrease in nasal resistance was observed in the natural state and tubeassisted modes from $\mathrm{T} 1$ to $\mathrm{T} 2$, but differences were not significantly different for the spray-assisted or sprayand tube-assisted modes. From T1 to T3, there were highly significant differences observed for all experimental conditions except for the spray- and tube-assisted mode. See Fig. 7.

C. Comparison of each subgroup with the control group. Comparison of resistance values for the control group at $\mathrm{Tl}$ with the "lo" expansion subgroup at T1 showed a significant difference only with the tube-assisted mode (the control group actually had a higher median resistance value than the "lo" subgroup with the tubes). There were no significant differences between the resistance values for the control group at $\mathrm{T} 1$ and the "lo" subgroup following expansion (T2) or at the 9- to 12-month follow-up (T3).

Comparison of resistance values for the control group at T1 with the "hi" expansion group at T1 showed significant differences for the natural state and tubeassisted modes, but no significant differences for the spray-assisted and spray- and tube-assisted modes. Resistance values were not significantly different between the control group at $\mathrm{T} 1$ and the "hi" subgroup following maximum expansion (T2) for all experimental modes. However, at T3 resistance values for the "hi" expansion subgroup continued to be significantly higher than those for the control group for the natural state and tubeassisted modes.

$V$. Comparison of the boys versus girls. Within each time period, there was no significant difference in nasal resistance values between the boys and girls in either the expansion or control groups. However, differences were apparent when time periods were compared for each group separately. Only the girls showed a significant decrease from $\mathrm{T} 1$ to $\mathrm{T} 2$ for the natural state, whereas the group of boys demonstrated a significant decrease only from $T 1$ to $T 3$. Neither the boys nor girls, when considered separately, showed significant de-

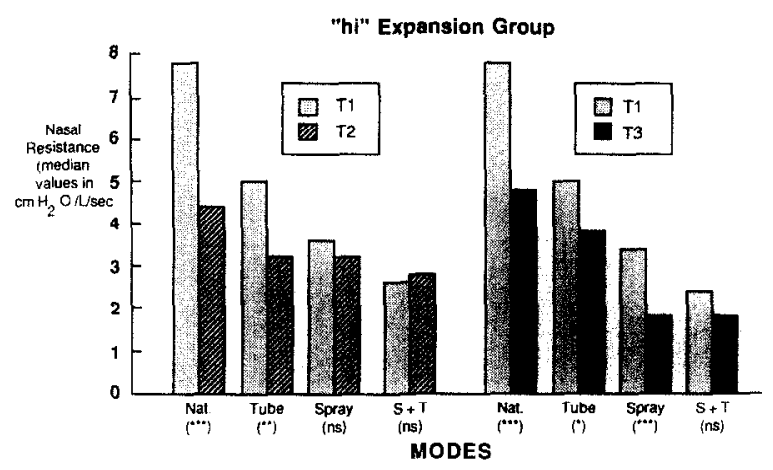

Fig. 7. Median nasal resistance for the "hi" expansion subgroup: $\mathrm{T} 1$ to $\mathrm{T} 2, \mathrm{~T} 1$ to $\mathrm{T} 3$.

creases in nasal resistance for the spray-assisted mode from T1 to T2. Note: data for all subjects are available in Hartgerink. ${ }^{20}$

\section{DISCUSSION}

Our results indicate that RME may decrease nasal resistance in some persons and, on average, this effect appears to be stable within a 1-year period. It has been suggested that growth and development may also have an effect on decreasing nasal resistance. The findings showed a slight but insignificant median decrease in nasal resistancc for the natural state, but a significant decrease for the spray-assisted mode in the control group from $\mathrm{T} 1$ to $\mathrm{T} 3$, which may be attributable to the effects of maturation.

Age-related decreases in nasal resistance (of approximately $0.1 \mathrm{~cm} \mathrm{H} 20 / \mathrm{L} / \mathrm{sec}$ per year) have previously been reported by other authors. ${ }^{21.22}$ Growth does appear to decrease nasal resistance, but over a 1-year period this effect is very small. These findings are supported by Melsen, ${ }^{23}$ who has shown internal resorption of the bony nasal cavity occurring up to age 15 . This involves growth of the facial skeleton with accompanying increases in width and area of the nasal cavity, and may be a factor contributing to a decrease in nasal resistance. The high forces resulting from rapid maxillary expansion probably also induce remodeling of the bones of the nasal cavity in addition to that occurring with growth. ${ }^{24}$ Atrophy of lymphoid tissue during development is also thought to contribute to decreases in nasal resistance. ${ }^{25}$

\section{Effect of rapid maxillary expansion and experimental conditions on nasal resistance}

Comparison of the expansion group with the control group showed that patients receiving RME for orthodontic purposes had a much higher median nasal resistance than those not receiving RME. The decrease in 
nasal resistance in the expansion group from $\mathrm{T} 1$ to $\mathrm{T} 2$ (Fig. 1) indicates that, on the average, RME contributes to reductions in nasal resistance. However, individuals do not necessarily respond according to group norms and a high degree of inter-subject variation was evident. Approximately $35 \%$ of the sample either increased or stayed approximately the same from $\mathrm{T} 1$ to $\mathrm{T} 2$ for the natural state, indicating that RME did not affect their nasal resistance. Part of this variation may be due to the transient effects of the soft tissues of the nasal cavity and includes cyclic swelling of nasal mucosa, nasal polyps, mucosal hyperplasia, allergic rhinitis, and infectious swelling of the nasal cavity. ${ }^{26-28}$

Two subgroups could be identified within the expansion group. One subgroup ("lo") showed little change in nasal resistance values following RME, whereas the other subgroup ("hi") showed substantial decreases in basal resistance following RME. Those persons who showed little change, or even increased nasal resistance, tended to have much lower nasal resistance values in the natural state initially (generally less than or equal to $5.5 \mathrm{~cm} \mathrm{H} 20 / \mathrm{L} / \mathrm{sec}$ ) than those who showed decreased nasal resistance following expansion.

It is interesting to note that this value $(5.5 \mathrm{~cm}$ $\mathrm{H} 20 / \mathrm{L} / \mathrm{sec}$ ) was found to be compatible with predominantly nasal breathing as quantified with the SNORT apparatus. Several persons were identified who demonstrated $80 \%$ to $100 \%$ nasal breathing despite a nasal resistance of $5.5 \mathrm{~cm} \mathrm{H} 20 / \mathrm{L} / \mathrm{sec}$. A separate study testing associations between nasal resistance and oral/nasal ratios has now been completed. ${ }^{29}$ Our findings disagree with the contentions of Watson, Warren, and Fischer. ${ }^{10}$ They classified nasal resistance as high if greater than or equal to $4.5 \mathrm{~cm} \mathrm{H20/L} / \mathrm{sec}$ and low if less than that value (measured at flow of $0.5 \mathrm{~L} / \mathrm{sec}$ ). Their "critical" value of $4.5 \mathrm{~cm} \mathrm{H} 20 / \mathrm{L} / \mathrm{sec}$ was selected because they believed that "clinically observable mouth breathing is more consistent above this level than below it." However, their assessment of "mouth breathing" involved only a subjective evaluation by an otorhinolaryngologist. In our study, by means of the SNORT apparatus, we quantified oral/nasal airflow ratios and confirmed predominantly $(>80 \%)$ nasal breathing at resistance values of $5.5 \mathrm{~cm} \mathrm{H} 20 / \mathrm{L} / \mathrm{sec}$.

The much greater and highly significant reduction in nasal resistance of the "hi" expansion subgroup, as compared with the "lo" subgroup between T1 and T2, seems primarily attributable to RME. Resistance decreases of such magnitude cannot be ascribed to growth and development alone because no such changes occurred in the control group.
The traditional explanation for the influence of RME on nasal resistance is based on the lateral separation of the walls of the nasal cavity, which occurs concurrently with dental arch expansion. Increasing the distance between the lateral walls of the nasal cavity and the nasal septum may decrease nasal resistance by enlarging the cross-sectional area of the nasal passage to facilitate breathing. This may be similar to the effect seen with growth and development, but with a more rapid and immediate effect. Binasal width (between the lateral walls of the nasal cavity) was not measured in this study. However, Turbyfill ${ }^{16}$ could not demonstrate a significant correlation between decreases in nasal resistance during expansion and increases in lower binasal width, and concluded that the various other factors that determine nasal resistance obscure any demonstrable linear correlation.

In his study of RME, Wertz ${ }^{30}$ showed that the greatest expansion occurs at the lower anterior portion of the nasal cavity. Consequently, he maintained that nasal airway conductance would not be increased by rapid maxillary expansion if an obstruction was not in this area, but further posteriorly.

An additional explanation for the effect of RME in producing decreases in nasal resistance should also be considered. Rapid maxillary expansion may have a similar effect on nasal resistance as does dilation of the anterior nares produced by placement of tubing for some patients. For the expansion group, the use of tubes placed in the anterior nares at $\mathrm{T} 1$ decreased the nasal resistance to that of the control group in the natural state at $\mathrm{T} 1$. Resistance with tubing at $\mathrm{T} 1$ was also similar to the values obtained in the natural state following maximum expansion at T2 (Figs. 1 and 3 ).

Increasing nasal conductance with dilation of the anterior nares with Tygon tubing was also obtained by Berkinshaw. ${ }^{31}$ Similar decreases in nasal resistance were demonstrated by Turvey, Hall, and Warren, ${ }^{32}$ following superior maxillary surgical impaction. They found that the alar base tended to be widened with a "flaring" of the nares postsurgically. They suggested that the decreases in nasal resistance observed may have been caused by dilation of the nares and an opening of the liminal valve (the point of greatest constriction at the anterior nares).

Our findings suggest that some persons with high nasal resistance may have airway constriction at the anterior nares, rather than posterior to this area. Such constrictions may be normalized (or deconstricted) following expansion of the anteroinferior aspect of the nasal cavity. It is noteworthy that only the girls showed significant decreases in nasal resistance from $\mathrm{T} 1$ to $\mathrm{T} 2$. 
We do not know if girls in general tend to have a smaller cross-sectional area than do boys at the anterior nares or whether this finding was limited to our sample.

The use of vasoconstrictive nose drops was advocated by Linder-Aronson and Backstrom ${ }^{28}$ to lessen the effect of mucosal swelling mainly at the anterior aspect of the inferior turbinates. This was recommended to give a better indication of the effect of the bony structures of the nasal cavity upon nasal resistance. Nasal resistance would thus be determined more by anatomic (skeletal), rather than function (soft-tissue), influences following decongestant administration and would emphasize the contribution of the pharyngeal component to the total nasophyaryngeal resistance. Linder-Aronson and Aschan ${ }^{5}$ found significant decreases in nasal resistance after expansion when recording nasal resistance with nasal decongestant. In their view, maxillary expansion normalized the anatomic condition of the nose by decreasing soft-tissue influences and improving "nasal function" to a normal range.

Our study, however, did not show significant decreases in nasal resistance following expansion with the use of the decongestant. If the decongestant mode does more accurately represent the anatomic influences and changes occurring, and if expansion causes a significant change in the width of the nasal cavity by reducing a possible obstructing effect, then this should be reflected in the comparisons between $\mathrm{T} 1$ and $\mathrm{T} 2$ for the sprayassisted mode. Yet the differences that we noted with this protocol were insignificant, suggesting that maxillary expansion has little or no effect on nasal airway resistance. This is in accord with the conclusions of Wertz and Dreskin. ${ }^{4}$

\section{Individual response to expansion and mode of testing}

The preceding explanations for treatment responses may not be adequate to explain the entire range of individual variation. As with recordings in the natural state, individual variation was also high for the other three modes. For example, anatomic variations in the shape of the anterior nares may also explain differences due to the placement of Tygon tubes. A person may have long, thin anterior nares, perhaps even with a "collapsed" appearance, and thus show a definite expansion with Tygon tubes in place, resulting in a reduction in nasal resistance.

Other patients may have circular-shaped anterior nares, exhibit little change after insertion of the Tygon tubes, with no resultant effect on nasal resistance, and possibly even experience an obstructive effect with an increased nasal resistance. In general, if the inner di- ameter of the tube was the same or smaller than the lumen of the anterior nares, then nasal resistance could be unaffected or increased following placement. If the Tygon tubes increased the effective diameter of the nares, nasal resistance might also be unaffected if the minimum cross-sectional area was posterior to the anterior nares.

Nasal resistance also decreased in many subjects following the use of the decongestant because this reduced soft-tissue swelling. Slight additional decreases were sometimes seen with the placement of tubes at the antcrior nares after decongestant use, suggesting that an obstruction was present due to both anatomic structures (nares construction) and functional structures (soft-tissue swelling). Other subjects showed increased nasal resistance with the addition of the Tygon tubes, indicating an obstructive effect at the anterior nares.

In contrast, several other subjects showed essentially no change in nasal resistance from the natural state with the use of tubes or decongestant. This may indicate that the location of minimum cross-sectional area is in the posterior region of the nasal cavity and therefore unaffected by either anterior nares dilation or vasoconstriction of the nasal mucosa in the anterior aspect of the nasal cavity.

\section{Clinical implications}

Testing with different experimental conditions may aid in the determination of the location of an obstruction contributing to increased nasal resistance. For example, if Tygon tubes placed at the anterior nares significantly reduced nasal resistance, then the obstruction may be present at the anterior nares. If decongestant significantly reduced nasal resistance, the obstruction may be determined by soft-tissue influences in the anterior aspect of the nasal airway. If clinically indicated, turbinate reduction, septoplasty, or allergy therapy may help reduce nasal resistance in some persons; however, such procedures will not benefit patients with a maximum airway constriction located at the anterior nares.

Adenoidectomy would be of no benefit to those persons demonstrating a constriction at the anterior nares or anterior aspect of the nasal cavity. Adenoids influence the posterior regions of the nasal airway and do not contribute to increases in nasal resistance anteriorly. However, if nasal resistance is high, but is unaffected by either placement of Tygon tubing or the use of a decongestant, an obstruction contributing to increased nasal resistance may be located at the posterior aspects of the nasopharynx. In such cases hypertrophied adenoidal tissue may well be the major contributor to increased nasal resistance. 


\section{CONCLUSIONS}

The following conclusions were derived from this study.

1. For studies of resistance to be comparable, the same flow rates and experimental conditions of testing must be used.

2. The range of individual variation for nasal resistance was high for all four test conditions. Median response for the group was not an adequate estimate of individual response. Extrapolation from group medians to individuals is an unsound practice and may be clinically misleading.

3. Some patients receiving RME for orthodontic reasons had a significantly greater nasal resistance to airflow before expansion than the control group. This was highly variable, however, as many patients in the expansion group actually had initial resistance values lower than those of the control group.

4. There was a significant median decrease in nasal resistance for the group after undergoing RME in the natural state only. This, however, was significant only for the subgroup consisting of subjects with initial resistance values greater than $5.5 \mathrm{~cm} \mathrm{H} 20 / \mathrm{L} / \mathrm{sec}$. Resistance decreases attributable to RME appeared to be stable up to 1 year after maximum expansion.

5. Significant reductions in nasal resistance were not found between $\mathrm{T} 1$ and $\mathrm{T} 2$ when pressure/flow data were obtained after the administration of decongestant spray.

6. RME may effect an expansion at the anterior nares contributing to reductions in nasal resistance.

7. Statistical significance does not necessarily equate with clinical relevance. Many of the statistically significant changes seen for the group were within the range of individual variation.

8. Because of the high variability in individual response, RME is not a predictable means of decreasing nasal resistance.

9. As with any treatment, a risk factor/benefit ratio should be evaluated before recommending RME. The benefit is in terms of gaining space for orthodontic correction. The potential risk is related to the stability or relapse of such treatment. As reduction in nasal resistance has yet to be unequivocally proved as a significant factor that improves subsequent growth, the recommendation of RME for purely respiratory reasons cannot be advocated on a risk/benefit basis.

The authors would like to thank Dr. James McNamara for providing many of the patients for this study. We are indebted to Dr. Charles Kowalski for his advice on statistical issues.

\section{REFERENCES}

1. Derischweiler H. Gaumennahteraeitervng. Munich: Karl Hanser, 1956.

2. Korkhaus G. Present orthodontic thought in Germany: jaw widening with active appliances in cases of mouth breathing. AM J ORTHOD 1960;46:187-206.

3. Haas AJ. Rapid expansion of the maxillary dental arch and nasal cavity by opening the midpalatal suture. Angle Orthod 1961; 31:73-90.

4. Wertz R, Dreskin M. Midpalatal suture opening: a normative study. AM J ORTHOD 1977;71:367-81.

5. Linder-Aronson S, Aschan G. Nasal resistance to breathing and palatal height before and after expansion of the median palatal suture. Odont Revy 1963;14:254-70.

6. Subtelny JD. Oral respiration: facial maldevelopment and corrective dentofacial orthopedics. Angle Orthod 1980;50:147-64.

7. Bresolin D, Shapiro PA, Shapiro GG, Chapko MK, Dassel S. Mouth breathing in allergic children: its relationship to dentofacial development. AM J ORTHOD 1983;83:334-40.

8. Huber RE, Reynolds JW. A dentofacial study of male students at the University of Michigan in the physical hardening program. AM J ORTHOD ORal SuRu 1946;32:1-21.

9. Leech HA. Clinical analysis of orofacial morphology and behavior of 500 patients attending an upper respiratory clinic. Dent Pract Dent Rec 1958;9:57-68.

10. Watson R, Warren D, Fischer N. Nasal resistance, skeletal classification, and mouth breathing in orthodontic patients. $\Lambda \mathrm{M} J$ ORTHOD 1968;54:367-79.

11. Vig PS, Sarver DM, Hall DJ, Warren DW. Quantitative evaluation of nasal airflow on relation to facial morphology. AM J ORTHOD 1981;79:263-72.

12. Warren DW. Aerodynamic studies of upper airway: implications for growth, breathing, and speech. In: McNamara J, ed. Nasorespiratory function and craniofacial growth. Monograph 9. Ann Arbor, Michigan: 1979. Center for Human Growth and Development, University of Michigan.

13. Timms DJ. Rapid maxillary expansion. Chicago: Quintessence Publishing Co., 1981:118.

14. Wertz RA. Changes in nasal airflow incident to rapid maxillary expansion. Angle Orthod 1968;38:1-11.

15. Hershey HG, Stewart BL, Warren DW. Changes in nasal airway resistance associated with rapid maxillary expansion. AM J ORTHOD 1976;69:274-84.

16. Turbyfill WJ. The long-term effect of rapid maxillary expansion on nasal airway resistance [Master's thesis]. University of North Carolina, 1976.

17. Gurley WH, Vig PS. A technique for the simultaneous measurement of nasal and oral respiration. AM J ORTHOD 1981;82: 33-41.

18. Keall C, Vig PS. An improved technique for the simultaneous measurement of nasal and oral respiration. AM J ORTHOD [in press, 1986].

19. Warren D. A quantitative technique for assessing nasal airway impairment. AM J ORTHOD 1984;86:306-14.

20. Hartgerink DV. The effect of rapid maxillary expansion on nasal airway resistance: a one year follow-up [Master's thesis]. Ann Arbor, Michigan: University of Michigan, 1986.

21. Warren DW, Duany LF, Fischer ND. Nasal pathway resistance in normal and cleft lip and palate subjects. Cleft Palate $\mathbf{J}$ 1969;6:134-40.

22. Principato JJ, Wolf P. Pediatric nasal resistance. Laryngoscope 1975;95:1067-9. 
23. Melsen B. Palatal growth studied on human autopsy material: a histologic microradiographic study. AM J ORTHOD 1975;68: 42-54.

24. Walters RD. Facial changes in the Macaca mulatta monkey by orthopedic opening of the midpalatal suture. Angle Orthod 1975;45:169-79.

25. Massler M, Zwemer JD. Mouthbreathing. II. Diagnosis and treatment. J Am Dent Assoc 1953;46:658-71.

26. Stoksted $P$. Measurements of resistance in the nose during respiration at rest. Acta Otolaryngol 1953(suppl 109):143-58.

27. Ballard CF, Gwynne-Evans E. Discussion of the mouth breather. Proc R Soc Med Sect Paed 1958;51:279-85.

28. Linder-Aronson S, Backstrom A. A comparison between mouth and nose breathers with respect to occlusion and facial dimensions. Odont Revy 1960;11:343-76.

29. Keall HJ. The relationship between nasal resistance and respi- ratory mode [Master's thesis]. Ann Arbor, Michigan: University of Michigan, 1986.

30. Wertz RA. Skeletal and dental changes accompanying rapid midpalatal suture opening. AM J ORTHOD 1970;58:41 66.

31. Berkinshaw ER. Pressure-flow studies for nasal inspiration and expiration [Master's thesis]. Chapel Hill, North Carolinạ: University of North Carolina, 1981.

32. Turvey TA, Hall DJ, Warren DW. Alterations in nasal airway resistance following superior positioning of the maxilla. AM J ORTHOD 1984;85:109-14.

Reprint requests to:

Dr. Peter S. Vig

Department of Orthodontics

The University of Michigan

Ann Arbor, MI 48109 